\title{
LMI-based Control Design to Enhance Robustness of Synchronous Power Controller
}

\author{
Ngoc Bao Lai \\ Loyola University Andalusia \\ Loyola.Tech \\ Seville, Spain \\ nblai@uloyola.es
}

\author{
Andrés Tarrasó \\ Universitat Politecnica de Catalunya \\ SEER (UPC) \\ Terrassa, Spain \\ andres.tarraso@upc.edu
}

\author{
Pedro Rodriguez \\ Loyola University Andalusia, \\ Universitat Politecnica de Catalunya \\ Loyola.Tec, SEER (UPC) \\ Spain \\ prodriguez@uloyola.es
}

\begin{abstract}
Synchronous power controller (SPC) has emerged as a suitable technique to equip grid-connected inverters with grid supporting functionalities such as inertial emulation and frequency/voltage support by mimicking the behavior of synchronous machines. Although the feasibility of the SPC has been experimentally verified under various operating conditions, parameter tuning for the SPC to ensure a stable inverter system has not been adequately addressed in the literature. To fill this gap, this paper presents a robust control design for the SPC to ensure its stable operation under the grid impedance variation. The proposed design procedure consists of system modelling and robust optimal parameter selection by using linear matrix inequality approach. The effectiveness of the proposed control design is proven by means of simulations and experiments.
\end{abstract}

Keywords - linear matrix inequality, synchronous power controller, virtual admittance, virtual synchronous machine.

\section{INTRODUCTION}

Recently, virtual synchronous machine concept for inverter-based distributed generators has drawn the attention from many researchers from industry and academia [1], [2]. As a result, several control schemes have been proposed in the literature [3]. Among the widely used approaches, the synchronous power controller (SPC) has been proved to be an attractive solution due to its simplicity and effectiveness [4], [5]. Since the SPC consists of several cascaded control loops, it is therefore necessary to design these loops properly not only to comply with grid codes but also to maintain a stable operation of the inverter system under different grid conditions.

Considering the power control loop of the SPC, a flexible droop characteristics was proposed in [6]. In this work, the active power controller is designed to contain one zero and one pole instead of only on pole as in the conventional SPC. This modification gives rise to one more degree of freedom for the power control loop. As a result, damping factor and the inherent droop slope can be independently configured. Likewise, multiple virtual admittance based control approach was presented in [7] to reduce the harmonic content in injected currents. In this study, the virtual admittance is specifically designed for each harmonic sequence. Such improvement allows the SPC to control the harmonic content of the injected current. To take into account of all control loops, [8] proposed a generalized formulation for designing the SPC. Even though different aspects in parameter design of the SPC was tackled in the aforementioned proposals, variation in grid impedance which is the main source of instability was not taken into account.

Considering the grid impedance variation, this paper presents a robust optimal design procedure for the SPC. The proposed control scheme emphasizes on the electrical part of

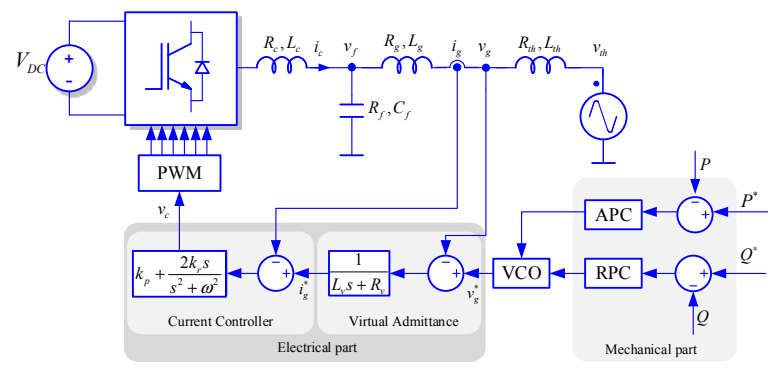

Fig. 1. Block diagram of the conventional SPC.

the SPC where the adverse influence of LCL-filter takes place. The main contributions of this paper are the derivation of a state-space model of the electrical part and the utilization of linear matrix inequality (LMI) approach for selecting robust optimal gains. Comparative simulation and experimental results are provided to validate the proposed control scheme.

The remaining of this paper is organized as follow: the section II presents the derivation of the SPC model by using state-space equations. From the system model, the robust optimal gains are calculated in the section III. Finally, the simulation and experimental results are presented in the section IV.

\section{MODELLING OF THE SPC}

Fig. 1 shows the block diagram of the conventional SPC which consists of an electrical part and a mechanical part. The mechanical part being composed of an active power controller (APC) and reactive power controller (RPC) emulates the electromechanical interaction of a synchronous machine. On the other hand, the electrical part including a virtual admittance block and a current controller imitates the electromagnetic interaction of a synchronous machine. Since these two parts have different bandwidths, their dynamics are nearly decoupled. Therefore, their parameters can be independently tuned to meet stability and performance criteria. Since the selection of the parameters for the mechanical part has already addressed in detail in [6], this paper mainly focuses on the design of the electrical part. In this regard, the $L C L$-filter can be modelled in $\alpha \beta$-frame as

$$
\begin{gathered}
\dot{\mathbf{x}}_{l c l c}(t)=\mathbf{A}_{l c l c} \mathbf{x}_{l c l c}(t)+\mathbf{B}_{l c l c} u(t)+\mathbf{G}_{l c l c} w(t) \\
y_{l c l}(\mathrm{t})=\mathbf{C}_{l c l} \mathbf{x}_{l c l c}(\mathrm{t})
\end{gathered}
$$

where $\mathbf{x}_{l c l c}=\left[\begin{array}{lll}i_{c} & v_{f} & i_{g}\end{array}\right]$ are the inverter-side current, capacitor voltage, and grid-side current, respectively, $u=v_{c}$ 
is the inverter input voltage, $y=i_{g}$, and the system matrices are as follows:

$$
\begin{gathered}
\mathbf{A}_{l c l c}=\left[\begin{array}{ccc}
-\frac{R_{c}}{L_{c}} & -\frac{1}{L_{c}} & 0 \\
\frac{1}{C_{f}}-\frac{R_{c} R_{f}}{L_{c}} & -\frac{R_{f}}{L_{g}+L_{t h}}-\frac{R_{f}}{L_{c}} & R_{f} \frac{R_{g}+R_{t h}}{L_{g}+L_{t h}}-\frac{1}{C_{f}} \\
0 & \frac{1}{L_{g}+L_{t h}} & -\frac{R_{g}+R_{t h}}{L_{g}+L_{t h}}
\end{array}\right] \\
\mathbf{B}_{l c l}=\left[\begin{array}{c}
\frac{1}{L_{c}} \\
\frac{R_{f}}{L_{c}} \\
0
\end{array}\right], \mathbf{G}_{l c l c}=\left[\begin{array}{c}
0 \\
\frac{R_{f}}{L_{g}+L_{t h}} \\
-\frac{1}{L_{g}+L_{t h}}
\end{array}\right], \mathbf{C}_{l c l}=\left[\begin{array}{l}
0 \\
0 \\
1
\end{array}\right]
\end{gathered}
$$

where $R_{c}, R_{f}$, and $R_{g}$ are the filter resistances, $L_{c}$ and $L_{g}$ are the filter inductances, $C_{f}$ is the filter capacitance, and $R_{t h}$ and $L_{t h}$ are the resistance and the inductance of grid impedance. It is worth noting that grid impedance is usually represented by SCR and $q=X / R$ ratio as

$$
\begin{gathered}
R_{t h}=\frac{V^{2}}{S C R \cdot P_{n} \sqrt{1+q^{2}}} \\
L_{t h}=\frac{V^{2} q}{S C R \cdot P_{n} \cdot \omega_{g} \sqrt{1+q^{2}}}
\end{gathered}
$$

with $V, P_{n}$, and $\omega_{g}$ being line to line grid voltage, nominal power of the inverter, and nominal grid angular frequency

For the purpose of designing a digital control system, (1) and (2) can be discretized as follows:

$$
\begin{gathered}
\mathbf{x}_{l c l}(k+1)=\mathbf{A}_{l c l} \mathbf{x}_{l c l}(k)+\mathbf{B}_{l c l} u(k)+\mathbf{G}_{l c l} w(k) \\
y_{l c l}(k)=\mathbf{C}_{l c l} \mathbf{x}_{l c l}(k) .
\end{gathered}
$$

The delay caused by the digital implementation can also be modeled as follows:

$$
\begin{gathered}
{\left[\begin{array}{c}
x_{l c l}(k+1) \\
x_{d}(k+1)
\end{array}\right]=\left[\begin{array}{cc}
\mathbf{A}_{l c l} & \mathbf{B}_{l c l} \\
\mathbf{0} & 0
\end{array}\right]\left[\begin{array}{c}
x_{l c l}(k) \\
x_{d}(k)
\end{array}\right]+\left[\begin{array}{c}
\mathbf{0} \\
1
\end{array}\right] u(k)+\left[\begin{array}{c}
\mathbf{G}_{l c l} \\
0
\end{array}\right] w(k)} \\
y_{l c l}(k)=\left[\begin{array}{ll}
\mathbf{C}_{l c l} & 0
\end{array}\right]\left[\begin{array}{c}
\mathbf{x}_{l c l}(k) \\
x_{d}(k)
\end{array}\right]
\end{gathered}
$$

where $x_{d}$ is a dummy state that presents the one sampling step delay.

Equation (7) and (8) can be rewritten in a compact form as

$$
\begin{gathered}
x_{i n}(k+1)=\mathbf{A}_{i n} x_{i n}(k)+\mathbf{B}_{i n} u_{i n}(k)+\mathbf{G}_{i n} w_{i n}(k) \\
y_{i n}(k)=\mathbf{C}_{i n} \mathbf{x}(k)
\end{gathered}
$$

\section{Proposed Control Scheme}

Considering the electrical part of the SPC, while the virtual impedance should be tuned properly to meet the grid code requirement, the current controller can be arbitrary designed provided that it can track references. For modeling the virtual admittance, the following equation is used.

$$
i_{g}^{*}(t)=-\frac{R_{v}}{L_{v}} i_{g}^{*}(t)+\frac{1}{L_{v}} v_{g}^{*}(t)-\frac{1}{L_{v}} v_{g}(t)
$$

where $R_{v}$ is the virtual resistance, $L_{v}$ is the virtual inductance, $i_{g}^{*}$ is the reference current for current controller, and $v_{g}^{*}$ is reference voltage coming from power control loop. The discrete-time counterpart of (11) is

$$
x_{v a}(k+1)=A_{v a} x(k)+\mathrm{B}_{v a} v_{g}^{*}(k)-\mathrm{B}_{v a} v_{g}(k)
$$

The current controller is designed by using internal model principle as

$$
\dot{\mathbf{x}}_{p r c}(t)=\left[\begin{array}{cc}
0 & 1 \\
-\omega_{g}^{2} & 0
\end{array}\right] \mathbf{x}_{p r c}(t)+\left[\begin{array}{l}
1 \\
0
\end{array}\right] e(t)
$$

or in a compact form as

$$
\dot{\mathbf{x}}_{p r c}(t)=\mathbf{A}_{p r c} \mathbf{x}_{p r c}(t)+\mathbf{B}_{p r c} e(t)
$$

where $\omega_{g}$ is the fundamental grid frequency. Equation (14) can be discretized as

$$
\mathbf{x}_{p r}(k+1)=\mathbf{A}_{p r} \mathbf{x}_{p r}(k)+\mathbf{B}_{p r} e(k)
$$

Equation (9), (12), and (15) can be augmented as

$$
\mathbf{x}(k+1)=\mathbf{A x}(k)+\mathbf{B} u(k)+\mathbf{G} v_{t h}(k)+\mathbf{H} v_{g}^{*}(k)(16)
$$

where

$$
\begin{aligned}
& \mathbf{A}=\left[\begin{array}{ccc}
\mathbf{A}_{i n} & \mathbf{0} & \mathbf{0} \\
-\mathbf{B}_{p r} \mathbf{C}_{i n} & \mathbf{A}_{p r} & \mathbf{B}_{p r} C_{v a} \\
B_{v a} \mathbf{H}_{v g} & \mathbf{0} & A_{v a}
\end{array}\right], \mathbf{B}=\left[\begin{array}{c}
\mathbf{B}_{i n} \\
\mathbf{0} \\
0
\end{array}\right], \mathbf{G}=\left[\begin{array}{c}
\mathbf{G}_{i n} \\
\mathbf{0} \\
B_{v a} M_{v g}
\end{array}\right], \\
& \mathbf{H}=\left[\begin{array}{lll}
\mathbf{0} & \mathbf{0} & B_{v a} M_{v g}
\end{array}\right]^{T}, M_{v g}=L_{g} /\left(L_{g}+\mathrm{L}_{t h}\right), \text { and } \\
& \mathbf{H}_{v g}=\left[\begin{array}{ll}
0 & 1-L_{g} /\left(L_{g}+\mathrm{L}_{t h}\right) \quad L_{g}\left(R_{g}+\mathrm{R}_{t h}\right) /\left(L_{g}+\mathrm{L}_{t h}\right)-R_{g} \quad 0
\end{array}\right]
\end{aligned}
$$

The system in (16) is stable if and only if the following inequality holds. 


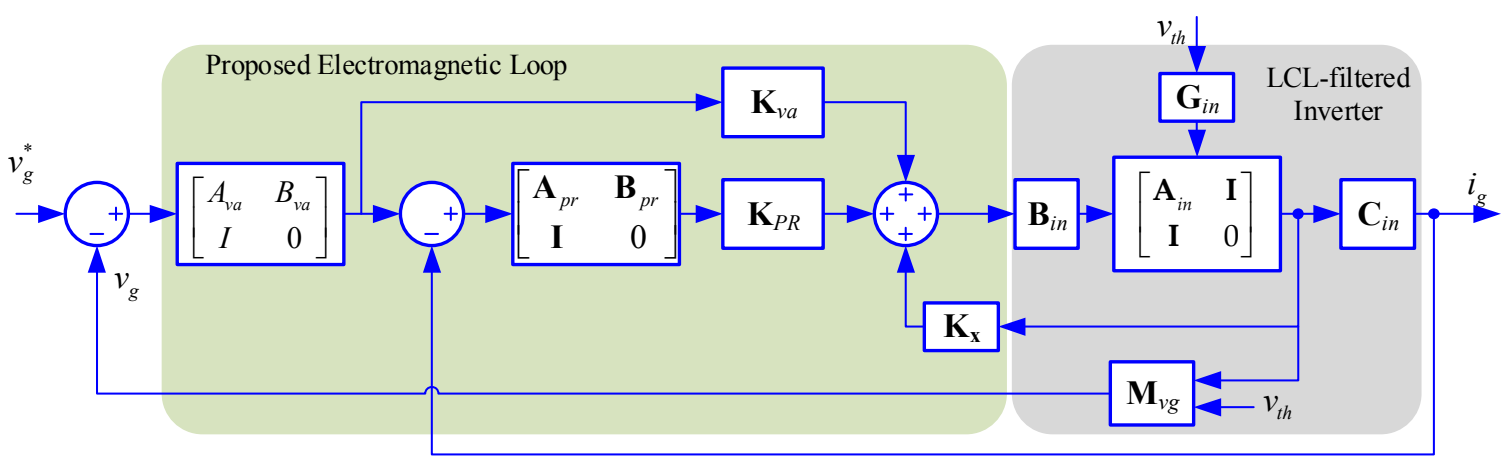

Fig. 2. The proposed control scheme.

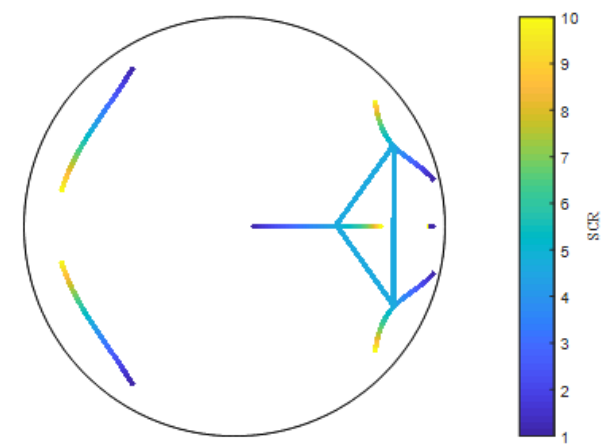

(a)

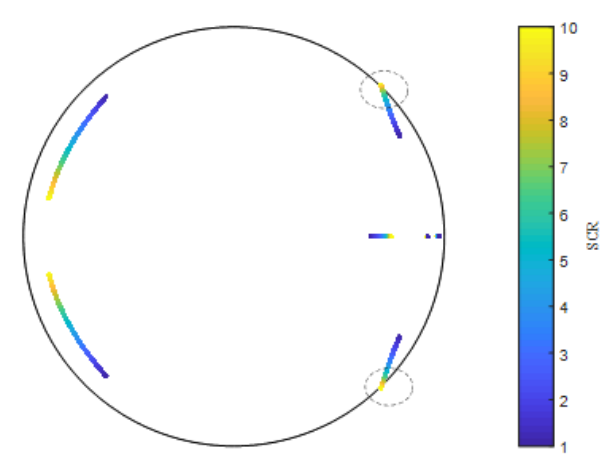

(b)

Fig. 3. Eigenvalues of the conventional SPC; (a) Current control loop only. (b) Electrical loop including the virtual admittance and current control loop.

$$
\left[\begin{array}{cc}
\varepsilon^{2} \mathbf{Q} & \left(\mathbf{A}_{i} \mathbf{Q}+\mathbf{B}_{i} \mathbf{Y}\right)^{T} \\
\left(\mathbf{A}_{i} \mathbf{Q}+\mathbf{B}_{i} \mathbf{Y}\right)^{T} & \mathbf{Q}
\end{array}\right]>0 \quad \text { and } \quad \mathbf{Q}>0
$$

where $\mathbf{Q}=\mathbf{R}^{-1}$ with $\mathbf{R}$ being a positive definite matrix with appropriate dimension, and $\mathbf{Y}=\mathbf{K Q}$ with $\mathbf{K}$ being the feedback gain vector.

To find a robust optimal feedback gain, the following LMI optimization [9] is employed.

$$
\underset{\varepsilon}{\operatorname{Minimize}} \mathbf{Q} \text { subject to (17) }
$$

TABLE I. SYSTEM PARAMETERS

\begin{tabular}{lcc}
\hline Inverter nominal power: $P$ & 350 & $\mathrm{~kW}$ \\
Grid nominal line voltage & 360 & $\mathrm{~V}$ \\
Grid nominal frequency & 50 & $\mathrm{~Hz}$ \\
DC-link voltage & 800 & $\mathrm{~V}$ \\
\hline Inverter-side resistance & 2.4 & $\mathrm{~m} \Omega$ \\
Inverter-side inductance & 125 & $\mu \mathrm{H}$ \\
Capacitor resistance & 25 & $\mathrm{~m} \Omega$ \\
Filter capacitance & 300 & $\mu \mathrm{F}$ \\
Grid-side resistance & 1 & $\mathrm{~m} \Omega$ \\
Grid-side inductance & 50 & $\mu \mathrm{H}$ \\
\hline Virtual resistance & 53 & $\mathrm{~m} \Omega$ \\
Virtual inductance & 509 & $\mu \mathrm{H}$ \\
\hline
\end{tabular}

where $\varepsilon$ is the convergent speed of the state which is tunable parameter determining the bandwidth of the closed-loop system, subscript $i=1,2$ indicate that the system matrix and the input matrix is evaluated at the maximum and minimum value of short circuit ration (SCR). The proposed control scheme is depicted in Fig. 2.

\section{SIMULATION AND EXPERIMENTAL RESULTS}

To validate the proposed control scheme, comparative simulations and hardware-in-the-loop (HIL) tests have been carried out. The system parameters are given in Table I. For the HIL tests, the inverter and filters are simulated in real-time simulator while the controller is implemented in a DSP-based control board. For both simulations and HIL tests, the switching frequency and sampling frequency are set at 3.15 $\mathrm{kHz}$ and $6.3 \mathrm{kHz}$, respectively.

To illustrate the limitation of the conventional SPC, Fig. 3 shows the eigenvalue maps of the SPC with and without virtual admittance loop. It can be seen from Fig. 3 a that the as the SCR increases the system become more and more stable when only current controller is used. However, when the virtual admittance is introduced, the system become unstable as the SCR reaches 10 . As a consequence, it can be concluded that introducing the virtual admittance loop may deteriorate the stability of the inverter system. Therefore, it is necessary to consider the virtual admittance when designing the current controller.

Fig. 4a shows that the proposed electrical part has the same gain and phase delay with those of the conventional one. Thus, steady-state response of the proposed electrical part is the same with that of the conventional one despite their structural differences. However, by considering the variation of SCR in 


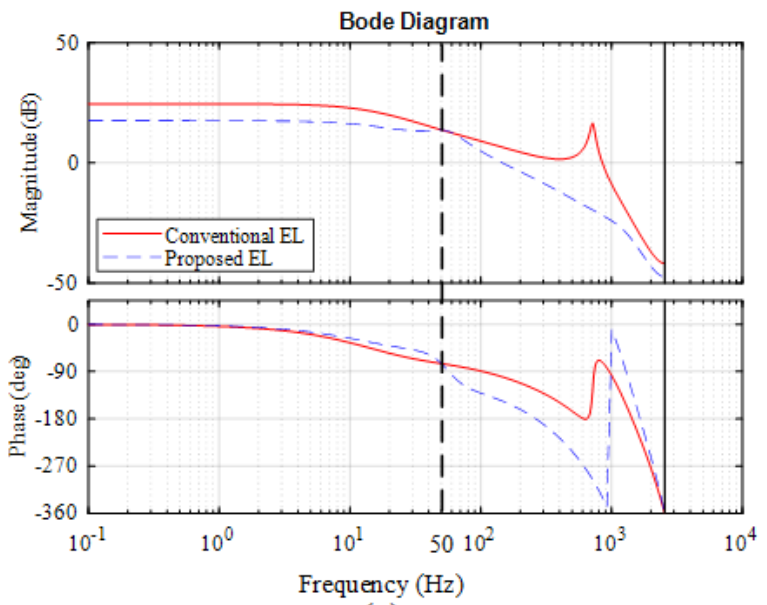

(a)

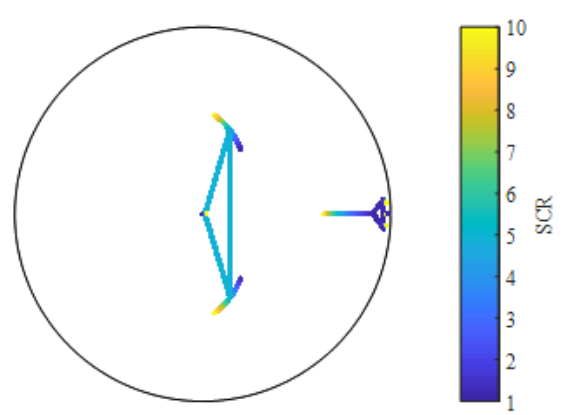

(b)

Fig. 4. (a) Frequency response of the conventional electrical part and the proposed electrical part. (b) Eigenvalue map of the improved SPC.

controller design, the stability of the proposed electrical part is ensured regardless of the variation in SCR as clearly shown in Fig. 4b.

Fig. 6 shows the simulation responses of the conventional electrical part and the proposed electrical part. As seen, due to a step change in SCR value of the grid at $t=0.5 \mathrm{~s}$, the conventional SPC becomes unstable resulting in highly distorted current waveform. On the contrary, the response of the proposed electrical part is unaffected by the variation of SCR. This confirms the effectiveness of the proposed control design approach.

To verify the proposed control approach in a practical system, the HIL is used as shown in Fig. 6. The power converter is simulated in the real-time simulator while the controller is implemented in the DSP-based control board. As shown in Fig. 7, the HIL results are well consistent with those in simulation.

\section{CONCLUSION}

It has been shown that introducing virtual admittance loop might reduce the stability margin of the inverter system due to the fact that the dynamics of the virtual admittance and the current controller are coupled. This paper has presented a controller design methodology to tackle with the aforementioned limitation of the conventional SPC. The
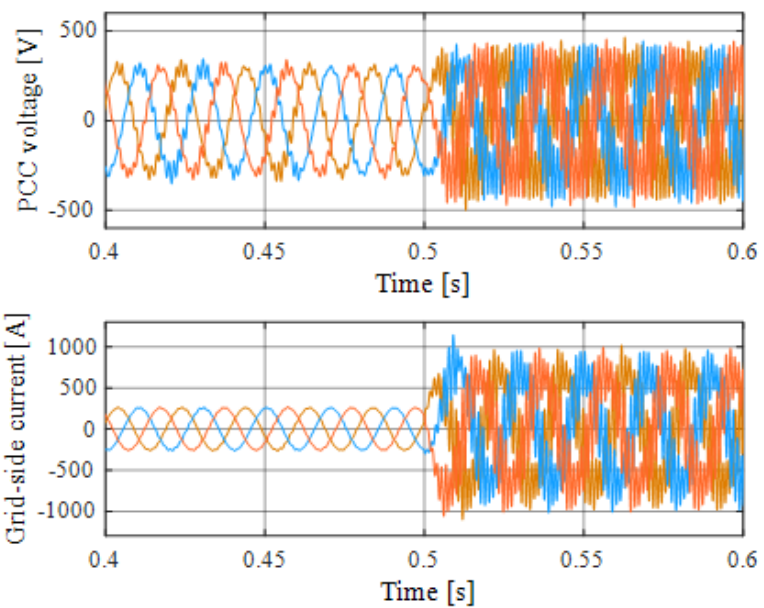

(a)
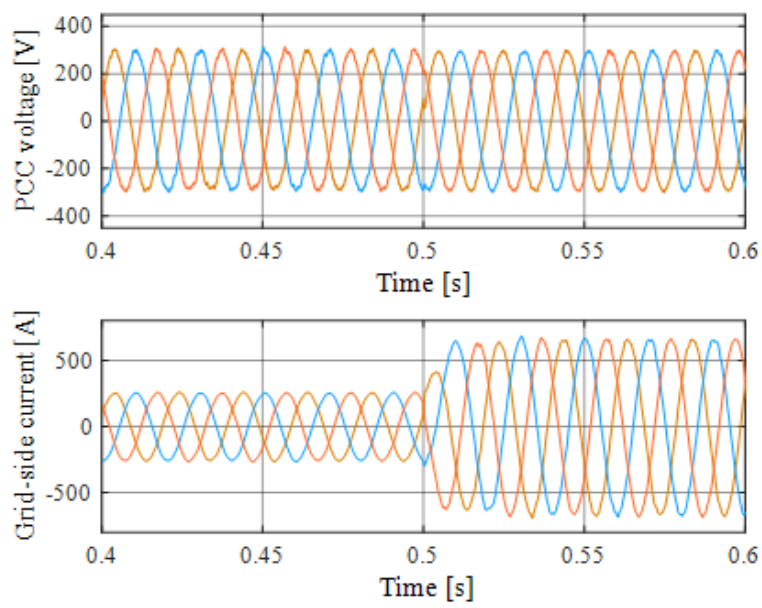

(b)

Fig. 5 Simulation results of the electrical part under a step change of SCR at $\mathrm{t}=0.5 \mathrm{~s}$; (a) Conventional electrical part. (b) Proposed electrical part.

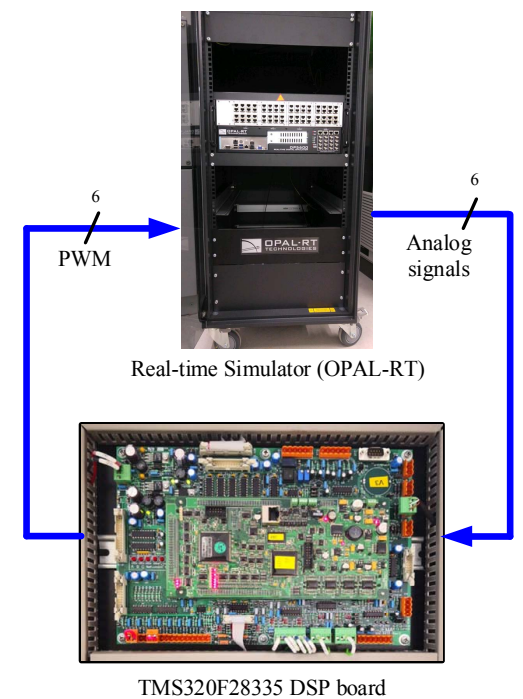

Fig. 6. Hardware-in-the-loop setup.

proposed control scheme focuses on the electrical part of the SPC. To considering the variation of the grid SCR, the state- 


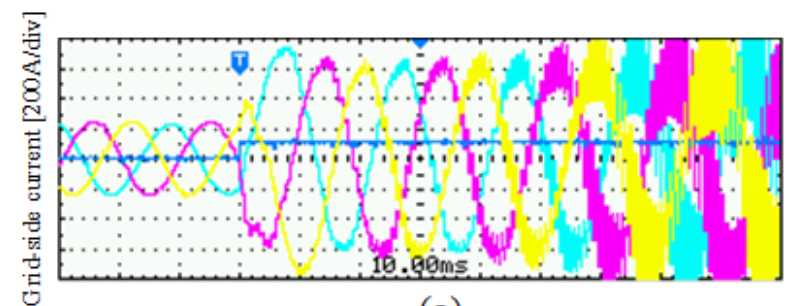

(a)

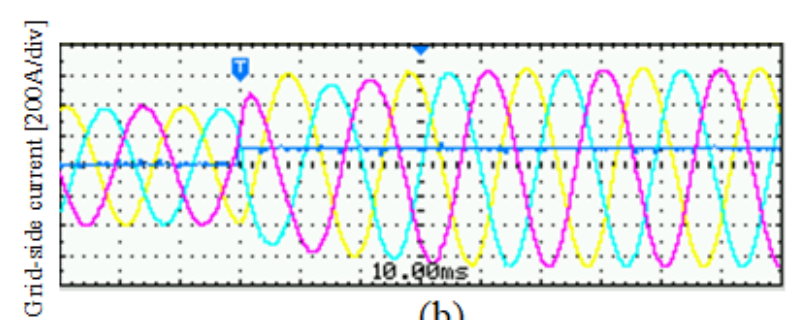

(b)

Fig. 7 HIL verification under a step change of SCR (indicated by a step change in the blue waveform); (a) Conventional electrical part. (b) Proposed electrical part.

space model of the SPC is used. By applying the LMI approach, the proposed control scheme can ensure a stable operation of the inverter regardless of the variation on the grid SCR. Simulation and experimental results have been provided to verify the effectiveness of the proposed control scheme.

\section{ACKNOWLEDGMENT}

This work was supported by the European Commission under the project FLEXITRANSTORE - H2020-LCE-20162017-SGS-774407 and by the Spanish Ministry of Science Innovation and Universities under project SMARTNODES ENE2017-88889-C2-1-R.

\section{REFERENCES}

[1] H.-P. Beck and R. Hesse, "Virtual synchronous machine," in 9th International Conference on Electrical Power Quality and Utilisation, Barcelona, 2007.

[2] S. D'Arco, J. A. Suul and O. B. Fosso, “A virtual synchronous machine implementation for distributed control of power converters in smartgrids," Electric Power Systems Research, vol. 122, no. 1, pp. 180-197, 2015.

[3] S. D'Arco and J. A. Suul, "Virtual synchronous machines - classification of implementations and analysis of equivalence to droop controllers for microgrids," in IEEE Grenoble Conference, Grenoble, 2013.

[4] P. Rodriguez, J. I. Candela, J. Rocabert and R. Teodorescu, "Synchronous power controller for a generating system based on static power converters". World Patent WO2012117131A1, 28022011.

[5] D. Remon, A. M. Cantarellas, J. M. Mauricio and P. Rodriguez, "Power system stability analysis under increasing penetration of photovoltaic power plants with synchronous power controllers," IET Renewable Power Generation, vol. 11, no. 6, pp. 733-741, 2017.

[6] W. Zhang, A. M. Cantarellas, J. Rocabert, A. Luna and P. Rodriguez, "Synchronous power controller with flexible droop characteristics for renewable power generation systems," IEEE Transactions on Sustainable Energy, vol. 7, no. 4, pp. 1572 - 1582, 2016.

[7] A. Tarrasó, J. I. Candela, J. Rocabert and P. Rodriguez, "Grid voltage harmonic damping method for SPC based power converters with multiple virtual admittance control," in IEEE Energy Conversion Congress and Exposition (ECCE), Cincinnati, 2017.

[8] R. Rosso, S. Engelken and M. Liserre, "A generalized formulation of active power synchronization based control algorithms for grid connected converters," in IECON 2018 - 44th Annual Conference of the IEEE Industrial Electronics Society, D.C, 2018.

[9] N.-B. Lai and K.-H. Kim, "Robust control scheme for three-phase grid-connected inverters with LCL-filter under unbalanced and distorted grid conditions," IEEE Transactions on Energy Conversion, pp. 506 - 515, June 2018 . 